\title{
Atypical mycobacterial infection of the lung in rheumatoid arthritis
}

\author{
S W WATKIN, ${ }^{1}$ R C BUCKNALL, ${ }^{1}$ M NISAR, ${ }^{2}$ AND R A L AGNEW \\ From the ${ }^{1}$ Medical Unit, Broadgreen Hospital, Liverpool and the ${ }^{2}$ Chest Unit, Fazakerley Hospita $\overbrace{\triangleright}$ \\ Liverpool
}

SUMMARY Mycobacterium kansasii was isolated from a cavitating pneumonia found in a 51 yeå old man with seropositive rheumatoid arthritis, and treatment was complicated by drug induced neuropathy.

Key words: Mycobacterium kansasii, upper lobe cavitation.

Patients with rheumatoid arthritis are prone to develop a variety of pleuropulmonary complications, including pleurisy with or without effusion, fibrosing alveolitis, nodules, and obstruction of airways. There is also an increased incidence of infection..$^{1-3}$ More recently it has been suggested that upper lobe cavitation may be regarded as a distinct pulmonary complication of rheumatoid disease.$^{45}$ Tuberculosis is found more commonly in patients with rheumatoid arthritis than in those with other forms of arthritis. ${ }^{67} \mathrm{We}$ report here a case of atypical mycobacterial infection of the lung causing upper lobe cavitation in a patient with rheumatoid arthritis.

\section{Case report}

In March 1986 the patient, a 51 year old male Caucasian retired police officer, attended for routine follow up at a rheumatology clinic complaining of a dry cough of two months' duration and weight loss of $13 \mathrm{~kg}$. He smoked 20 cigarettes a day. His arthritis had been quiescent for about two years and his symptoms were well controlled by treatment with fenoprofen $600 \mathrm{mg}$ three times a day and diclofenac retard $100 \mathrm{mg}$ at night.

Six years previously the patient had been diagnosed as having rheumatoid arthritis, and initial

Accepted for publication 5 August 1988.

Correspondence to Dr S W Watkin, Medical Unit, Broadgreen Hospital, Thomas Drive, Liverpool L14 3LB. investigation had shown this to be a typical seroposi tive, erosive form of the disease. In addition, a ches radiograph at that time showed a fine bilatera放 pulmonary infiltrate compatible with diffuse pul $\stackrel{\mathbb{Q}}{\square}$ monary fibrosis. There were no symptoms referablet to this and no further action was taken at that time The arthritis was initially difficult to control withp non-steroidal anti-inflammatory agents alone, an treatment with gold was therefore started. This wast continued for two and a half years when $D \bar{Q}$ penicillamine was substituted. The patient stopped this of his own accord in December 1985 after an episode of vomiting. The patient has never received systemic steroids.

On examination there was a nodular deforming rheumatoid arthritis. There was no clubbing oD lymphadenopathy. In the chest there was dullness to. percussion over the right upper lobe with coarse inspiratory crackles and a pleural friction rub over the same area. A fever of $38^{\circ} \mathrm{C}$ was noted.

A chest radiograph showed the previously noted bilateral fine infiltrate, the appearances of whicts were unchanged and, in addition, an area of consolidation in the right upper lobe containingo multiple cavities (Fig. 1). Tomography of the area showed no additional features but confirmed the presence of a cavitating pneumonia, which was felP to be tuberculous in appearance. Other investiga tions included a haemoglobin of $160 \mathrm{~g} / \mathrm{l}$, a polymor $\overline{\mathrm{Q}}$ phonuclear leucocytosis of $13 \times 10^{9} / 1$, and an erythrog cyte sedimentation rate (Westergren) of $37 \mathrm{~mm}$ ip 


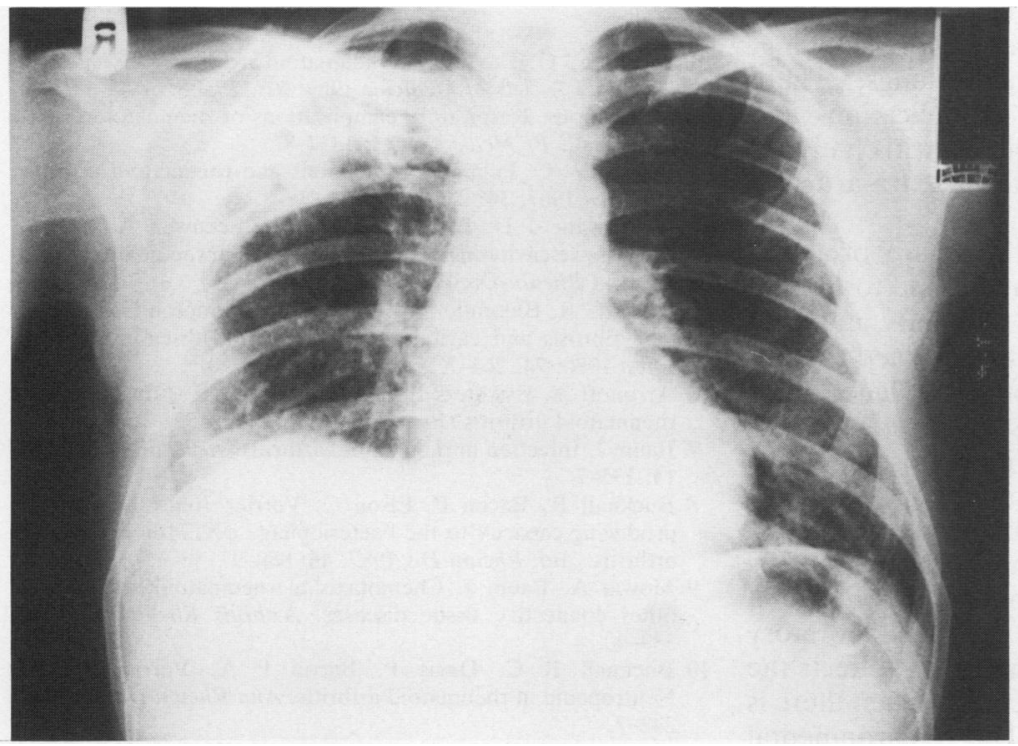

Fig. 1 Chest radiograph showing cavitating pneumonia right upper lobe and also bilateral basal pulmonary infiltrate.

the first hour. The patient had very little sputum production but this was negative on staining for acid/alcohol fast bacilli and grew only normal flora on routine culture. Sputum cytology was negative. A Mantoux test 1 in 1000 (10 TU) showed no reaction, and serology produced no significant antibody titres to influenza, respiratory syncytial virus, adenovirus, Coxiella burnetii, mycoplasma, or chlamydia. The latex fixation test for rheumatoid factor was positive and antinuclear antibodies were not detected. A full biochemical profile was normal. In view of the likelihood of tuberculous infection pulmonary function tests were not performed.

Treatment with broad spectrum antibiotics including anaerobic cover was started with amoxycillin $500 \mathrm{mg}$ and metronidazole $400 \mathrm{mg}$ both three times a day. There was clinical improvement. Tuberculosis was still suspected, however, and as no sputum samples were positive a fibre optic bronchoscopy was arranged. There was no endobronchial lesion and the bronchial mucosa appeared normal. Washings were taken from the right upper lobe and submitted for stain and culture for acid/alcohol fast bacilli.

Three weeks later in April 1986 the sputum grew an acid/alcohol fast bacillus, and treatment with the standard antituberculous regimen of rifampicin, isoniazid, and pyrazinamide was started pending identification of the bacillus. By this time the erythrocyte sedimentation rate had risen to $91 \mathrm{~mm}$ in the first hour, but the patient remained relatively well following the improvement seen with the aforementioned antibiotics. $\mathrm{He}$ has been well enough to allow home.
In June 1986 he attended the tuberculosis clinic complaining of jaundice and paraesthesia in the feet and hands. His chest radiograph was improved and the erythrocyte sedimentation rate had fallen to $50 \mathrm{~mm}$ in the first hour. By now the bacillus had been identified as Mycobacterium kansasii. These new symptoms were attributed to his drug treatment, and this was temporarily stopped. Pyridoxine $50 \mathrm{mg}$ three times a day was started on the assumption that the paraesthesia was due to isoniazid. Although the jaundice settled spontaneously, there was a deterioration in the neuropathy and he developed a severe disabling peripheral neuritis. Electromyography demonstrated an axonal peripheral neuropathy, which was predominantly sensory in type and compatible with isoniazid neuropathy. Symptomatic treatment with carbamazepine and amitriptyline resulted in some improvement.

Treatment for the mycobacterial infection was restarted with rifampicin and ethambutol, to which the bacillus had been shown to be sensitive, and by October 1986 there was continued improvement in the radiographic appearances. The patient had no respiratory symptoms and there was considerable improvement in his neuropathy. At the time of writing the patient remains well.

\section{Discussion}

The initial radiograph abnormality in this case was typical of diffuse pulmonary fibrosis and it is unusual that there were no relevant symptoms. It is known 
that once the chest radiograph has become abnormal it is likely there will be finger clubbing, dyspnoea, and basal crackles in the lungs. ${ }^{2}$ There had been no other pulmonary complications and after an initial difficult period the arthritis had also become quiescent. No other site of extra-articular disease was apparent.

Patients with rheumatoid arthritis are prone to opportunistic infection. This may be due to disease related, impaired cell mediated immunity and antibody producing capacity, ${ }^{8}$ or to the reduced chemotactic responses of polymorphonuclear leucocytes ${ }^{9}$ and the neutropenia of Felty's syndrome. ${ }^{10}{ }^{11}$ In addition, the use of steroids and immunosuppressive agents may increase the risk of infection. ${ }^{12}$

Among opportunistic mycobacteria in Britian $M$ kansasii may cause pulmonary disease and is an important member of environmental species. It can be identified at the Public Health Laboratory Service by its cultural characteristics. ${ }^{13}$ It infects the lungs of middle aged men, particularly when there is pre-existing lung damage. ${ }^{14}$ The environmental source of the organism is unknown, and person to person transmission rarely occurs.

In addition to pulmonary infection, $M$ kansasii has been reported to cause both infective polyarthritis and sporadic cases of tenosynovitis in patients with rheumatoid arthritis. ${ }^{15-17}$ These reports emphasised the point that lesions could have been mistaken for exacerbation of the underlying disease itself. This case is unusual in that the patient had not received either corticosteroid or immunosuppressant treatment. It seems likely that pulmonary infection with $M$ kansasii in this patient is related to defective immune mechanisms and perhaps asymptomatic pre-existing fibrosing alveolitis.

\section{References}

1 Scadding J G. The lungs in rheumatoid arthritis. Proceedings of the Royal Society of Medicine 1969; 62: 227-38.

2 Anonymous. Respiratory complications of rheumatoid diseas [Editorial]. Br Med J 1978; i: 1437-8.

3 Walker W C. Pulmonary infections and rheumatoid arthritis $Q J$ Med 1967; 36: 239-51.

4 MacFarlane J D, Franken C K, Van Leeuwen A W F M Progressive cavitating pulmonary changes in rheumatoid arthri tis. Ann Rheum Dis 1984; 43: 98-101.

5 Petrie G R, Bloomfield P, Grant I W, Crompton G K. Upper $\vec{O}$ lobe fibrosis and cavitation in rheumatoid disease. $\mathrm{Br} J \mathrm{Dis}-$ Chest 1980; 74: 264-7.

6 Arronoff A, Bywaters E G L, Fearnby G R. Lung lesions in rheumatoid arthritis. $\operatorname{Br}$ Med J 1955; ii: 228-32.

7 Baum J. Infection in rheumatoid arthritis. Arthritis Rhem $1971 ;$ 14: $135-7$.

8 Bucknall R, Bacon P, Elson C, Verrier Jones J. Antibody producing capacity to the bacteriophage $\phi X 174$ in rheumatoid arthritis. Ann Rheum Dis 1987; 46: 889-97.

9 Mowat A, Baum J. Chemotaxis in rheumatoid arthritis and other connective tissue diseases. Arthritis Rheum 1970; 13:음 $339-40$.

10 Bucknall R C, Davis P, Bacon P A, Verrier-Jones J.D Neutropenia in rheumatoid arthritis. Ann Rheum Dis 1982; 41:을. 242-7.

11 Sienknecht C W, Urowitz M B, Pruzanski W, Stein H B. Felty'sco syndrome. Clinical and serological analysis of 34 cases. Anno Rheum Dis 1977; 36: 500-7.

12 Ortbals D W, Marr J J. A comparative study of tuberculosis and other mycobacterial infections and their association with malig nancy. Am Rev Respir Dis 1978; 117: 39-45.

13 Wolinsky E. Non tuberculous mycobacteria and associated diseases. Am Rev Respir Dis 1979; 119: 107-59.

14 Grange J M, Yates M D. Infections caused by opportunistic mycobacteria. A review. $J R$ Soc Med 1986; 79: 226-9.

15 Demerieux P, Keystone E C, Hutcheon M, Laskin C. Poly $\overline{\bar{O}}$ arthritis due to Mycobacterium kansasii in a patient with rheumatoid arthritis. Ann Rheum Dis 1980; 39: 90-4.

16 Leader M, Revell P, Clarke G. Synovial infection withö Mycobacterium kansasii. Ann Rheum Dis 1984; 43: 80-2.

17 Glickstein S L, Nashel D J. Mycobacterium kansasii septic arthritis complicating rheumatic disease. A case report and. literature review. Arthritis Rheum 1987; 30: 231-5. 